\title{
Buchbesprechungen
}

Auschwitz-Prozess 4 Ks 2/63 Frankfurt am Main. Katalog zur Ausstellung. Herausgegeben von Irmtrud Wojak im Auftrag des Fritz Bauer Instituts, Gent (Snoeck Verlagsgesellschaft) 2004, 872 Seiten, $€ 46,98$.

Der bis dahin größte und umfassendste Prozess zur Aufarbeitung des Massenmords an den deutschen und europäischen Juden fand unter dem technischen Namen „Strafsache gegen Mulka und andere $4 \mathrm{Ks} 2 / 63$ " in den Jahren 1963 und 1965 in Frankfurt am Main statt. Nachdem insbesondere in den ersten Jahren der frühen Bundesrepublik kein politischer und juristischer Wille zur Aufarbeitung der nationalsozialistischen Gewaltherrschaft bestanden hatte, kam nach 1958, seit der Einrichtung der „Zentralen Stelle der Landesjustizverwaltungen zur Aufklärung nationalsozialistischer Verbrechen " in Ludwigsburg, wieder mehr Bewegung in die Strafverfolgung der NS-Verbrecher. Eine wesentliche Rolle spielte dabei der Namensgeber des Frankfurter Fritz Baner Instituts, der damalige hessische Generalstaatsanwalt. ${ }^{\mathrm{I}}$ Zum vierzigsten Jahrestag dieses tiefen Einschnitts in der bundesdeutschen Zeitgeschichte erarbeitete das Institut unter Federführung von Irmtrud Wojak eine Ausstellung, die über den Prozess und seine Rezeption in der deutschen Nachkriegsgesellschaft informiert. Ihre erste Station hatte sie im Frankfurter „Haus Gallus“, an historischem Ort: Hierhin war der Prozess nach der Eröffnung im Frankfurter Römer-Plenarsaal verlegt worden. Der Schwurgerichtssaal des Frankfurter Landgerichts war angesichts des großen Interesses der Medien und der Öffentlichkeit von Anfang an zu klein gewesen. Insgesamt nahmen ca. 20.000 Besucher am Prozess teil, darunter viele Schulklassen (258).

$\mathrm{Zu}$ dieser aufrüttelnden Ausstellung, die in der kommenden Zeit an verschiedenen Orten

I $\mathrm{Zu}$ Bauers wesentlichen politisch-juristischen Ansichten vgl. Fritz Bauer, Die Humanität der Rechtsordnung. Ausgewählte Schriften, hrsg. von Joachim Perels und Irmtrud Wojak (= Wissenschaftliche Reihe des Fritz Bawer Instituts, Band 5), Frankfurt am Main/New York s $99^{8}$.u in Deutschland und Israel zu sehen sein wird, ist ein umfangreicher Katalog erschienen, der ihre verschiedenen Elemente in Buchform aufbereitet. Der Natur eines Ausstellungskatalogs gemäß handelt es sich dabei nicht um ein Werk ,aus einem Guss“, sondern um eine Sammlung sehr unterschiedlicher Beiträge verschiedener Herangehensweisen: wissenschaftliche Aufsätze, Statistiken, Portraits der Angeklagten, Stimmen zur Rezeption des Prozesses in den Medien. Zahlreiche Illustrationen nutzen die Möglichkeiten eines Katalogs geschickt aus und erleichtern damit das Einfühlen in die damalige politische Stimmung.

Einen umfassenden Einstieg in die Thematik bieten vor allem die ersten drei wissenschaftlichen Aufsätze des Werkes von Hans Mommsen, Wolfgang Benz und Joachim Perels: Hans Mommsen (9I-IIO) bietet auf zwanzig Seiten eine konzentrierte Darstellung der Eckdaten der Judenverfolgung bis zur von den Nationalsozialisten so genannten „Endlösung“. Der komplizierte Weg zur Shoa wird nachgezeichnet und dabei die bis heute offenen Probleme der Forschung aufgezeigt: Einerseits ist offensichtlich, dass die deutsche Bevölkerungsmehrheit 1933 und später trotz z. T. massiver antisemitischer Einstellungen die eigentliche Judenverfolgung nicht wünschte und dann, als sie im Gange war, alles daran setzte, nicht mehr als nötig von ihr zu erfahren. Andererseits wurde das ungeheure Verbrechen, für das "Auschwitz“ exemplarisch steht, nach dem Scheitern anderer Projekte wie der vom Auswärtigen Amt und Reinhard Heydrich ersonnenen Abschiebung der Juden nach Madagaskar dann von "ganz normalen“ Deutschen kühl-rational und in diesem Sinne erbarmungslos durchgeführt. Dabei war es ein weiter Weg von der „eher visionären Perspektive“ (92), die Hitler selbst in Bezug auf die "Judenfrage" hatte, über die Pogrome von 1938, die Umsiedlung in das Generalgouvernement Polen und die 1 schon monströsen Verbrechen der SS-2und SD-Einsatzgruppen 
im Rücken der Front (98) und die WannseeKonferenz (104) bis hin zur Vergasung in Auschwitz (I06). Wesentlich bei dieser Schilderung ist und bleibt Mommsens klare Aussage, dass es eben nicht, wie nach dem Ende des Nationalsozialismus immer wieder behauptet, gelungen war, die fabrikmäßige Vernichtung von Menschenleben vor den Deutschen geheim zu halten, sondern dass zehntausende Funktionäre von SS, Polizei und Wehrmacht an der Exekution des Massenmordes beteiligt waren ( 107 ).

In einem zweiten Schritt befasst sich Wolfgang Benz, Direktor des Zentrums für Antisemitismusforschung an der TU Berlin, mit der Aufarbeitung der NS-Zeit in der frühen Bundesrepublik, erhellt also den politischkulturellen Hintergrund, vor dem der Auschwitz-Prozess stattfand: Treffend beleuchtet er den zwar ehrlichen, aber in seiner Art unpassenden, weil an wirklicher Klärung uninteressierten Antifaschismus Konrad Adenawers, der gegenüber unverbesserlichen Nazis lediglich „hausväterlichen Tadel“ zu bieten hatte und sie für ihre „ungezogenen Reden" kritisierte - um dann im nächsten Atemzug eine allgemeine Amnestie für Straftaten aus der Zeit des "Dritten Reiches“ ins Gespräch zu bringen. ${ }^{2}$ Benz geht auch auf die Entnazifizierung und auf die ersten juristischen Schritte zur Aufarbeitung ein, die dann von Joachim Perels (I24-I47) ausführlich bearbeitet wird. Perels schildert, wie schwer es für die Minderheit der Juristen war, die an der strafrechtlichen Ahndung der NS-Verbrechen interessiert war, sich gegen die Mehrheit durchzusetzen, für die das aus unterschiedlichen Gründen nicht galt - sei es aus eigener Kompromittierung, sei es aus falsch verstandener Kollegialität. Perels legt dar, wie schwierig es rechtspraktisch war, die im eigentlichen Wortsinne unfassbaren Verbrechen juristisch handhabbar zu machen, selbst wenn man prinzipiell für die Verfolgung eintrat. Hannab Arendts These wird angeführt, dass eine gerichtsförmige Aufarbeitung nicht möglich sei, weil die Ahndungsstruktur des Strafgesetzbuches einfach nicht auf den arbeitsteilig verübten Massenmord anwendbar sei. In einem Brief an Karl Jaspers schrieb sie 1946 mit resigniertem Unterton: „Göring zu hängen ist zwar notwendig, aber völlig inadäquat." (zit. I28) Diese vermeintliche Inadäquanz wurde natürlich von den Verteidigern - auch ohne Arendt gelesen zu haben - in der Weise aus-

2 Vgl. dazu den detaillierten Überblick in Norbert Frei: Vergangenheitspolitik. Die Anfänge der Bundesrepublik und die NS-Vergangenheit, München, 2. Auflage 1997. bes. Abschnitt I. genutzt, dass man sich für die Angeklagten auf den Befehlsnotstand berief (I 3 If.). Dagegen stellt Perels die Position von Claus Roxin, die dieser im Jahr des Beginns des Auschwitz-Prozess entwickelte: Die dialektische Beziehung zwischen Organisatoren und Exekutoren des Massenmords hebe individuelle Verantwortung keineswegs auf. Auch die Ausführenden - und um diese ging es im Prozess - sind nicht nur blindes Werkzeug weit entfernter anonymer Machthaber, sondern bleiben frei und verantwortlich für das, was sie tun. „Wer die Tat eigenhändig ausführt, ist Täter. Wer aber in einem Organisationsapparat an irgendeiner Stelle in der Weise eingeschaltet ist, daß er untergebenen Personen Befehle erteilen kann, ist ebenfalls Täter, wenn er seine Befugnis zur Durchführung strafbarer Handlungen einsetzt." (zit. I 30$)^{3}$ Leider wurde diese Position in den Prozessen selten konsequent angewendet. Vielmehr wurde die Stellung der Exekutoren des Massenmordes als vermeintliche „Rädchen im Getriebe“ fast durchgehend dazu verwendet, sie von Mördern zu bloßen Gehilfen zu machen. Bei aller grundsätzlichen Wertschätzung der Leistungen, die der Auschwitz-Prozess gebracht hat, insbesondere für die politische Kultur in Deutschland, bleibt mit Perels festzuhalten, dass das Urteil im Auschwitz-Prozess in derselben Tradition steht, die die Täter über Gebühr entlastet (I4I ff.; das Urteil 606 ff.).

Weitere wichtige Passagen des Katalogs, die sich spezifisch auf den Prozess beziehen, sind die Kurzbiographien der wichtigsten Angeklagten. Der Leser wird mit wichtigen Aussagen konfrontiert sowie Abdrucken von Dokumenten aus Auschwitz und weiteren Faksimiles, wodurch sich ein dreidimensionales Bild der Personen ergibt. Dazu gehören Unterschiede zwischen den Wahrnehmungen, die die Angeklagten von ihrer Rolle in Auschwitz haben wollten, und der, die sich durch Aussagen der Zeugen ergaben. Der höchstrangige Angeklagte, Robert Mulka, Adjutant des Lagerkommandanten Rudolf $H \ddot{o} \beta$, behauptete vor und während der Verhandlung steif und fest, er habe von den Morden nichts mitbekommen, habe niemals an der Rampe selektiert und wisse nichts von Vergasungen. Dagegen steht die Einschät-

3 So hatten es auch schon die meisten Juristen der Emigration gesehen, die für die Feststellung der Schuld neben den eigenen Taten auch die Stellung der Beklagten im Herrschaftssystem des NS-Systems heranziehen wollten. Vgl. dazu ausführlich Matthias Stoffregen, Kämpfen für ein demokratisches Deutschland. Emigranten zwischen Politik und Politikwissenschaft, Opladen 2002, 44 ff. In dieselbe Richtung zielte die Qualifizierung ganzer Orga2 nisationent als verbrecherisch durch den Nürnberger 4hauptkriegsverbrecherprozess. 
zung seines ehemaligen Vorgesetzten $H \ddot{ } \beta$ : „Der Adjutant ist der erste Gehilfe des Lagerkommandanten und steht $\mathrm{zu}$ ihm in einem besonderen Vertrauensverhältnis. Er hat dafür zu sorgen, daß dem Lagerkommandanten kein wichtiger Vorgang im Lager unbekannt bleibt.“ (zit. 293). Ähnlich Kopf schüttelnd bleibt der Leser zurück, wenn sich der Lagerarzt Dr. Capesius damit verteidigt, für ihn, der aus Siebenbürgen stammte, sei Deutschland immer ein „Muster an Ordnung und Gesetzmäßigkeit“ (346) gewesen, und deshalb habe er über Befehle und Verfahrensweisen nicht räsoniert, auch wenn sie ihm persönlich grausam erschienen seien. Kurz darauf liest man, wie Capesius eigenhändig herausgebrochene Zähne auf der Suche nach Goldfüllungen durchwühlt, um sich persönlich zu bereichern (352). Derlei Abscheulichkeiten werden dem Leser nicht erspart; genau so wenig übrigens dem Besucher der Ausstellung, der Tonbandmitschnitte des Prozesses anhören kann. Diese blieben eher zufällig erhalten und archiviert und werden erstmals der Öffentlichkeit zugänglich gemacht.

Wie bereits angedeutet, war der AuschwitzProzess über die Verhandlungen und die Urteile hinaus wichtig, weil er in bis dahin nicht gekanntem Umfang die deutsche Bevölkerung auf ihre Vergangenheit stieß und zur Auseinandersetzung zwang. Daher ist klar, dass eine Dokumentation des Prozesses seine Rezeption in den Medien mit umfasst. Der Katalog berichtet über achtzehn Personen, die im weitesten Sinne „Berichterstatter“ waren. Unter diesen Berichten waren sachliche Berichte dessen, was im Gerichtssaal verhandelt wurde, aber auch weitergehende Reportagen wie der Film „Bleiben die Mörder unter uns?" von Thilo Koch, in dem das persönliche Umfeld einzelner Angeklagter beleuchtet wurde - ihre eigene "heile Welt" (723 ff.). Besonders wichtig ist sicher die Berichterstattung Bernd Neumanns in der „Frankfurter Allgemeinen Zeitung“ gewesen (766 ff.), die eine wesentliche Vorlage für die dramatische Umsetzung des Prozessgeschehens in "Die Ermittlung" von Peter Weiss gewesen ist ( $782 \mathrm{ff}$.).

Der Katalog bietet eine umfassende Dokumentation der Ausstellung selbst und eröffnet eine Menge zusätzlicher Einsichten, die auch bei einem noch so intensiven Besuch der Ausstellung aus Zeitmangel gar nicht zu gewinnen sein dürften. Eine ausführliche Bibliographie und eine umfassende Filmographie komplettieren das Gesehene weiter. Allerdings stellt sich gelegentlich die Frage, wer genau der Adressatenkreis des Werkes sein könnte. Für Historiker findet sich wenig
Neues. Und für Rechtswissenschaftler, die sich mit dem Thema der juristischen Aufarbeitung der nationalsozialistischen Verbrechen befassen wollen, kann der Katalog nur einen Einstieg bieten; für ein genaues Quellenstudium reicht er naturgemäß nicht aus. An manchen Stellen wäre andererseits weniger mehr gewesen: Eine ausführliche Würdigung der Person Fritz Bawers kann in diesem Zusammenhang kaum gelingen, insofern könnten eine Reihe längerer Zitate aus seinen Schriften (8I6 ff.) hier weggelassen werden, zumal sie nicht kommentiert und mit dem Prozess selbst in Zusammenhang gebracht werden. Ebenfalls überflüssig sind Kurzportraits der Machthaber des Dritten Reichs wie Göring oder Goebbels. Die schon in der Ausstellung eher störenden künstlerischen Auseinandersetzungen mit der Thematik wirken auch im Katalog, in den sie an verschiedenen Stellen hineingestreut werden, etwas deplatziert.

Allerdings ist das Werk als Handreichung für Pädagogen und für Multiplikatoren der politischen Bildung äußerst empfehlenswert: Für jede Art der Annäherung an das Thema "Auschwitz" kann der Katalog wertvolle Anregungen bieten, gerade weil sein Inhalt so vielseitig und er keine Monographie ist. Insgesamt bildet er eine hervorragende und wichtige Ausstellung ab, für die dem Fritz Bawer Institut und besonders Irmtrud Wojak $\mathrm{zu}$ danken ist. Es bleibt $\mathrm{zu}$ hoffen, dass zumindest Teile der Ausstellung auch nach ihrem Ende der Öffentlichkeit zugänglich bleiben.

\section{Matthias Stoffregen}

Armin von Bogdandy (Hrsg.), Europäisches Verfassungsrecht. Theoretische und dogmatische Grundzüge, Berlin/Heidelberg (Springer Verlag) 2003, 978 Seiten, 59,95 $€$

Der Titel des in der Schlussphase des Europäischen Konvents 2002/2003 in einer Lehrbuchreihe erschienenen Werkes ist ambitioniert - der Verlag dürfte aus Marketinggründen auf ein so eingängiges Etikett Wert gelegt haben. Der Untertitel Theoretische und dogmatische Grundzüge wird dem Inhalt dieses voluminösen Sammelbandes besser gerecht. Das Buch umfasst 20 Beiträge von I9 Autoren und einer Autorin. Die einzige Autorin, die an der Queen's University in Belfast lehrende Politikwissenschaftlerin Antje Wiener, ist zugleich die einzige NichtJuristin im Autorenkreis. Die übrigen Autoren sind vorwiegend im Europarecht spezialisierte Juristen, die teils bereits zuvor durch ihr Interesse an interdisziplinären, über die 
Rechtsdogmatik hinausweisenden Fragen aufgefallen waren. „Verfassungsrecht ist denkbar ohne Staat, Nation und einen Akt, der sämtlichen Anforderungen des traditionellen Verfassungsbegriffs genügt." Diese plakative These aus dem Vorwort des Herausgebers legitimiert Konzeption und Inhalte des vorliegenden Bandes. Die theoretischen und dogmatischen Grundzüge eines europäischen Verfassungsrechts werden in i7 Einzelbeiträgen dargelegt und im zweiten Teil des Buches in drei „Gesamteinschätzungen“ zusammenfassend erörtert. Alle Beitragenden stammen aus dem deutschsprachigen Raum. Darin liegt zugleich ein zentrales Defizit des Buches, das einige der Autoren immerhin durch einen guten Überblick über die Fachdiskussionen im übrigen Europa und im Rest der Welt kompensieren. Insbesondere im englischsprachigen Raum und im Umfeld des Europäischen Hochschulinstituts in Florenz hat sich in den zurückliegenden Jahrzehnten eine umfangreiche Diskussion über die Funktionen des Rechts bei der europäischen Integration entwickelt. Ohne deren Beachtung wäre die deutschsprachige Diskussion der Gefahr ausgesetzt, in der lange Zeit dominierenden Blickverengung auf Konflikte zwischen dem europäischen Recht und dem mitgliedstaatlichen Verfassungsrecht zu verharren. Buch-Herausgeber Armin von Bogdandy hat an anderer Stelle die Tendenz zur selektiven Rezeption von Publikationen aus dem Sprachraum der jeweiligen Verfasser kritisch beleuchtet. ${ }^{\mathrm{I}}$ Auch der vorliegende Band hat dieses Problem nur teilweise überwunden.

Die Beiträge sind teils als Grundlagentexte mit starker theoretischer Fundierung, teils eher als kommentierende Zusammenfassung des rechtsdogmatischen Diskussionsstandes angelegt. Eine zentrale Bedeutung innerhalb des Werkes hat der erste, von Christoph Möllers verfasste Beitrag mit dem Titel Verfassungsgebende Gewalt - Verfassung Konstitutionalisierung, der sich mit dem Verfassungsbegriff im europäischen Kontext auseinandersetzt. Materialreich und fundiert analysiert Möllers die historischen Funktionen von herrschaftsbegründenden und herrschaftsbegrenzenden Verfassungen und ihre jeweiligen normativen Konnotationen (S. 4 ff.). Vor diesem Hintergrund verortet er die verschiedenen Strömungen der europäischen Verfassungsdiskussion, die zwischenzeitlich um die normativen Setzungen des Europäischen Konvents und der von ihm ausgearbeiteten Verfassung ergänzt worden

I A. von Bogdandy, Beobachrungen zur Wissenschaft vom Europarecht, in: Der Staat $200 \mathrm{I}, \mathrm{S}_{\frown 3}$ (7 ei passim $)$. sind. Ebenso interdisziplinär, grundlegend und hinsichtlich der internationalen Diskussion fundiert ist der Beitrag von Stefan Oeter zum Föderalismus (S. 59 ff.). Den Ausgangspunkt bildet eine Bestandsaufnahme der verschiedenen Strömungen der Föderalismusdebatte einschließlich der vehementen Ablehnung föderalistischer Konzepte für die europäische Integration in weiten Teilen des politischen und wissenschaftlichen Diskurses in Großbritannien. In Übereinstimmung mit dem Mainstream der heutigen Diskussion über die europäische Integration betont der Autor, dass die EG/EU durch die Herausbildung supranationaler Elemente verstärkt föderale Züge aufweist (S. 74). Ausführlich erörtert er zudem die Zusammenhänge der Föderalismusdiskussion mit Souveränitätsund Demokratietheorien.

Der Beitrag von Antje Wiener zum Thema Institutionen (S. I 2 I ff.) fällt aus dem Rahmen des Buches, indem er neuere Ansätze der politikwissenschaftlichen Institutionentheorie auf die europäische Integration anwendet und dabei über die „deutlich begrenzteren Interpretationen der deutschen Rechtswissenschaft" (S. I22) bewusst hinausgeht. Die Anwendung der verschiedenen Stränge politikwissenschaftlicher Institutionentheorie auf die Entwicklung der europäischen Integration und auf die neueren Konstitutionalisierungsprozesse liefert Material, das auch die rechtswissenschaftliche Diskussion sinnvoll ergänzt. Dies gilt z.B. für die Unterscheidung zwischen ,harten Institutionen“ (Organe der EG/EU) und „weichen Institutionen“ (Werte, Ideen, Handlungsroutinen).

Der Beitrag des Herausgebers mit dem Titel Europäische Prinzipienlehre arbeitet zutreffend heraus, dass es eine solche Prinzipienlehre bisher nur in Ansätzen gibt - stark geprägt von den Setzungen des Art. 6 des EUVertrags. Ebenso zutreffend betont er, dass die Herausbildung eines europäischen Verfassungsrechts sich nicht durch die einfache Übertragung von Prinzipien aus einzelnen nationalen Verfassungsordnungen bewerkstelligen lässt (S. I 59). In dem mit Souveränität und Vorrang überschriebenen Kapitel (S. 205 ff.) untersucht Alexander Schmitt Glaeser die Diskussionen über das Spannungsverhältnis zwischen mitgliedstaatlicher Souveränität und dem Anwendungsvorrang des Gemeinschaftsrechts - vorwiegend auf der Basis der EuGH-Rechtsprechung und der Kontroversen in der bundesdeutschen Fachdiskussion.

Der Aufsatz von Franz C. Mayer (S. 229 ff.) fragt nach dem Verhältnis zwischen mitgliedstaatlichen Verfassungsgerichten und dem 
EuGH. Damit basiert er implizit auf einem weiten Verfassungsbegriff, der anerkennt, dass der EuGH bereits heute verfassungsgerichtliche Funktionen wahrnimmt. Bei der vergleichenden Bestandsaufnahme der mit verfassungsgerichtlichen Aufgaben betrauten obersten mitgliedstaatlichen Gerichte nehmen die Funktionen des Bundesverfassungsgerichts und die diesbezügliche bundesdeutsche Diskussion allerdings wesentlich größeren Raum ein als die funktionsäquivalenten Gerichte der anderen Mitgliedstaaten. Die für die Entwicklung eines europäischen Verfassungsrechts besonders wichtige vergleichende Perspektive rückt auch in dem Beitrag von Christoph Grabenwarter über Staatliches Unionsverfassungsrecht (S. 283 ff.) in den Mittelpunkt, fokussiert insbesondere auf die verfassungsrechtlichen Bestimmungen der Mitgliedstaaten zu ihrem Verhältnis zur EG/EU. In unterschiedlicher Intensität werden hier alle is Mitgliedstaaten vor der Beitrittsrunde 2004 auf der Basis umfangreichen, über die juristische Literatur hinausreichenden Materials behandelt. Verschiedene Typologisierungsansätze liefern hier interessante Impulse für die weitere Diskussion, so z.B. die Unterscheidung zwischen integrationsoffenen und defensiven Anpassungen des mitgliedstaatlichen Verfassungsrechts an die europäische Integration (S. 333 f.).

Unter der Überschrift Völkerrechtliche $\mathrm{Ne}$ benverfassungen (S. 339 ff.) behandelt Robert Uerpmann zwei so unterschiedliche völkerrechtliche Regime wie die World Trade Organization (WTO) und die Europäische Menschenrechtskonvention (EMRK). Ihre in dem Beitrag herausgearbeitete Gemeinsamkeit besteht darin, dass sie jeweils spezifische Fragen bezüglich ihres Verhältnisses zu einem Verfassungsrecht der EU aufwerfen. Werner Schroeder behandelt mit den verfassungsrechtlichen Beziehungen zwischen der EU und der EG (S. 373 ff.) die unionsverfassungsrechtlichen Folgeprobleme der Entscheidung des Maastricht-Vertrags für einen Mix aus supranationaler und intergouvernementaler Integration im „Dreisäulensystem“ der EU. Bei einer erfolgreichen Ratifizierung der EU-Verfassung dürften sich diese Sonderprobleme im Wesentlichen erledigen.

Von grundlegender Bedeutung, wenn auch punktuell unter geänderten Vorzeichen, bleibt dagegen auch zukünftig die Kompetenzordnung im EU-System, die Martin Nettesheim ausführlich erörtert (S. 4I 5 ff.). Von bleibender Aktualität dürften auch die Ausführungen von Jürgen Bast zu den rechtlichen Handlungsformen der europäischen Integration sein, die in der neueren europap rechtlichen Literatur nur vergleichsweise selten ausführlicher behandelt worden sind auch wenn es mit dem Inkrafttreten der Verfassung zu einigen Umbenennungen und Differenzierungen der rechtlichen Handlungsformen kommen wird. Besonders interessant ist die historische Rekonstruktion des rechtswissenschaftlichen Interesses, auf das zunächst stärker die Verordnungen, später vor allem die Richtlinien in Abhängigkeit von den jeweils dominierenden Streitthemen der Rechtsprechung gestoßen sind.

In den folgenden Beiträgen rücken die individuellen Rechte der Menschen stärker in den Mittelpunkt, die sich in den I990er Jahren im Rahmen der Bemühungen um eine Abkehr von einer rein ökonomisch bestimmten Integration von einem Randthema zu einem Kernthema der europäischen Politik entwikkelt haben. Ein zentraler Aspekt ist hier die Unionsbürgerschaft, deren Entstehung, Bedeutung und Tragweite Stefan Kadelbach ausführlich erörtert (S. 539 ff.). Die Entwicklung europäischer Grundrechte in der Rechtsprechung des EuGH und der GrundrechteCharta fasst Jürgen Kübling zusammen (S. 583 ff.). Auch die offenen Fragen zum Verhältnis zwischen EU-Grundrechten und der Europäischen Menschenrechtskonvention werden in diesem Rahmen erörtert (S. 590 f. et passim). Den für die Wirtschaftsintegration konstitutiven Grundfreiheiten ist ein eigener, von Thorsten Kingreen verfasster Beitrag gewidmet (S. 63 I ff.), der nicht nur die einschlägige EuGH-Rechtsprechung übersichtlich systematisiert, sondern auch den politisch-institutionellen Kontext der Thematik anschaulich aufzeigt.

Die Beiträge von Armin Hatje (S. 683 ff.) und Josef Drexl (S. 747 ff.) zur Wirtschafts- und zur Wettbewerbsverfassung sind in Relation zu der zentralen Bedeutung der Wirtschaftsintegration als Kernelemente der Entwicklung der heutigen EG weit hinten in dem Buch platziert. Hatjes Bestandsaufnahme fasst die wirtschaftsverfassungsrechtlichen Elemente zusammen, die weite Teile des Primärrechts prägen. Er lotet zudem die Gestaltungsspielräume der europäischen Organe und der Mitgliedstaaten in diesem per definitionem marktwirtschaftlich konstituierten Bereich aus. Europäisches Wettbewerbsrecht als materielles Verfassungsrecht - der Untertitel von Drexls Aufsatz enthält die These, dass das Wettbewerbsrecht nicht nur zu den besonders praxisrelevanten Teilen des Gemeinschaftsrechts gehört, sondern auch von zentraler Bedeutung für ein europäisches Verfassungsrecht ist. Neben einer Auseinandersetzung mit dem Verhältnis zwischen eu- 
ropäischem Wettbewerbs- und Verfassungsrecht, das wiederum von dem verwendeten Verfassungsbegriff abhängt, enthält der Beitrag eine ausführliche Erörterung der dem Wettbewerbsrecht zugrunde liegenden wirtschaftstheoretischen Positionen und der einschlägigen EuGH-Rechtsprechung.

Der Beitrag von Ulrich Haltern mit dem Titel Gestalt und Finalität weist über die konventionellen verfassungsrechtlichen Fragestellungen hinaus, indem er Verfassungen kulturtheoretisch in ihrer Funktion als „Sinnspeicher" betrachtet. In dieser Perspektive fällt seine Bewertung des europäischen Verfassungsgebungsprojekts eher skeptisch aus.

In Anbetracht der thematischen Ausdifferenzierung sowie der Fülle an Diskussionsansätzen und Materialaufarbeitung in den I7 Beiträgen des Hauptteils, die in Summe mehr als 800 Seiten umfassen, verwundert es nicht, dass die drei abschließenden „Gesamteinschätzungen" kaum noch Neues zu bieten haben. Der Beitrag von Ulrich Everling beginnt mit einem historischen Überblick über die Entwicklung der europäischen Integration und ihrer rechtlichen Strukturen (S. 849 ff.), der allerdings über den Erkenntnisstand der vorliegenden integrationshistorischen Literatur nicht hinausgeht. Interessanter sind dagegen seine praxisnahen Einschätzungen als ehemaliger EuGH-Richter hinsichtlich der neueren Entwicklungen der europäischen Integration (S. 87 I ff.).

Der Aufsatz von Paul Kirchhof über die rechtliche Struktur der Europäischen Union als Staatenverbund (S. 893 ff.) lehnt sich eng an die von ihm in seiner Funktion als Berichterstatter in der Maastricht-Entscheidung des Bundesverfassungsgerichts (BVerfGE 89, I 55$)$ entwickelte Argumentation an. Die (begrenzte) Rezeption der Kategorie Staatenverbund in der internationalen Diskussion, ihre Anschlussfähigkeit und ihre möglichen Funktionen im weiteren Konstitutionalisierungsprozess bleiben dabei außer Betracht. In scharfem Kontrast zu Kirchhofs Beitrag, der sich dezidiert von den Bestrebungen zur Schaffung einer europäischen Verfassung abgrenzt, vertritt Manfred Zuleeg in seinem Schlussbeitrag (S. 93 I ff.) pointiert die Position, die EG/EU habe bereits eine Verfassung: „Das Vertragsrecht und das Richterrecht bilden die Verfassung der EU“ (S. 948).

Aufgrund des Patchwork-Charakters der Beiträge ist dieses Buch trotz oder gerade wegen seines großen Umfangs weit entfernt von einer geschlossen-systematischen Darstellung des sich entwickelnden europäischen Verfassungsrechts. An monographisch ange- legte Lehrbücher oder Arbeiten² kann der vorliegende Band insofern nicht heranreichen. Bisweilen stark divergierende Sichtweisen, unterschiedliche Maße an Internationalität und Überschneidungen an den Berührungspunkten zwischen den Beiträgen sind bei einem Sammelband mit sehr ausführlichen Einzelbeiträgen kaum zu vermeiden so bei der Einschätzung dessen, was eine Verfassung normativ und faktisch ausmacht, bei der föderalen Struktur oder der Bedeutung der Grundrechte, die in mehreren Beiträgen mehr oder minder ausführlich behandelt werden. Es fehlt auch eine über die alt bekannten Konflikte zwischen dem deutschen Verfassungsrecht und dem Gemeinschaftsrecht hinausweisende systematische Analyse der Defizite des gegenwärtigen europäischen Verfassungssystems in ihrer Konzentration auf die Wirtschaftsintegration, die sich auch durch die EU-Verfassung nicht grundlegend ändert.

Mehrere der Beiträge zeigen eine erfreuliche Offenheit der deutschsprachigen Europarechtswissenschaft für interdisziplinäre Sichtweisen, etwa wenn sie davon ausgehen, dass die Verfassungsentwicklung in der EU Bestandteil des Entwicklungsprozesses eines europäischen Mehrebenensystems ist. Die auf dieser Prämisse basierenden Beiträge zeigen zugleich, dass eine an der Verfassungswirklichkeit orientierte Dogmatik durch das Anknüpfen an analytische Modelle, die in der Politik- und Verwaltungswissenschaft auch international verwendet werden, integraler Bestandteil einer interdisziplinären Europaforschung sein kann.

Zukünftige Arbeiten zum europäischen Verfassungsrecht sollten stärker den Dialog mit den Sichtweisen der Verfassungsrechtswissenschaft anderer Mitgliedstaaten suchen, wie es auch in der Vergangenheit schon insbesondere Publikationen aus dem Umfeld des Europäischen Hochschulinstituts in Florenz getan haben. Auf die Sichtweise einer „Sprachfamilie“ beschränkte Herangehensweisen setzen sich dem Verdacht aus, die Positionen der eigenen Staatsrechtslehre in einer hegemonialen Konkurrenz gegen Positionen der verfassungsrechtlichen Europaforschung in anderen Mitgliedstaaten verteidigen $\mathrm{zu}$ wollen.

Hartmut Aden

2 So Koen Lenaerts/Piet van Nuffel, Constitutional Law of the European Union, London I999 (2. Aufl. 2005); Anne Peters, Elemente einer Theorie der Verfassung Europas, Berlin 200I; Thomas Giegerich, Europäische Verfassung und deutsche Verfassung im transnationalen Konstitutionalisierungsprozess: Wechselseitige Rezeption, konstitu2 tionelle Evolution und föderale Verflechtung, Heidelberg 